\title{
Molecular Study of L-Rhamnose Synthesis in Sporothrix schenckii and its Role in the Interaction with the Immune Innate System
}

Tamez-Castrellón A.K., López-Ramirez L.A., Mora-Montes H.M. Departamento de Biología, División de Ciencias Naturales y Exactas, Campus Guanajuato, Universidad de Guanajuato. Noria Alta s/n, Col. Noria Alta, Guanajuato, Gto. CP 36050, México. Tel Lab: (+52) 473-7320006 Ext. 8154. hmora@ugto.mx.

\section{INTRODUCTION}

Sporotrichosis is a mycosis of humans and mammals that has a worldwide distribution. This disease is caused by some of the members of the Sporothrix schenckii complex. S. schenckii is a dimorphic fungus that shows a mycelium morphology at $28^{\circ} \mathrm{C}$ and a yeast morphology at $37^{\circ} \mathrm{C}$. The first point of contact of the fungi with the cells of the host is the cell wall. A well know component of the cell wall of $S$. schenckii is the peptidorhamnomannan (PRM). This PRM is composed of $33.5 \%$ rhamnose and reacts with sera from patients with sporotrichosis. In bacteria, plants and fungi the L- Rhamnose is synthesized in similar ways. In bacteria the synthesis of this precursor involves four enzymes and in some fungi only three are needed (Fig. 1).

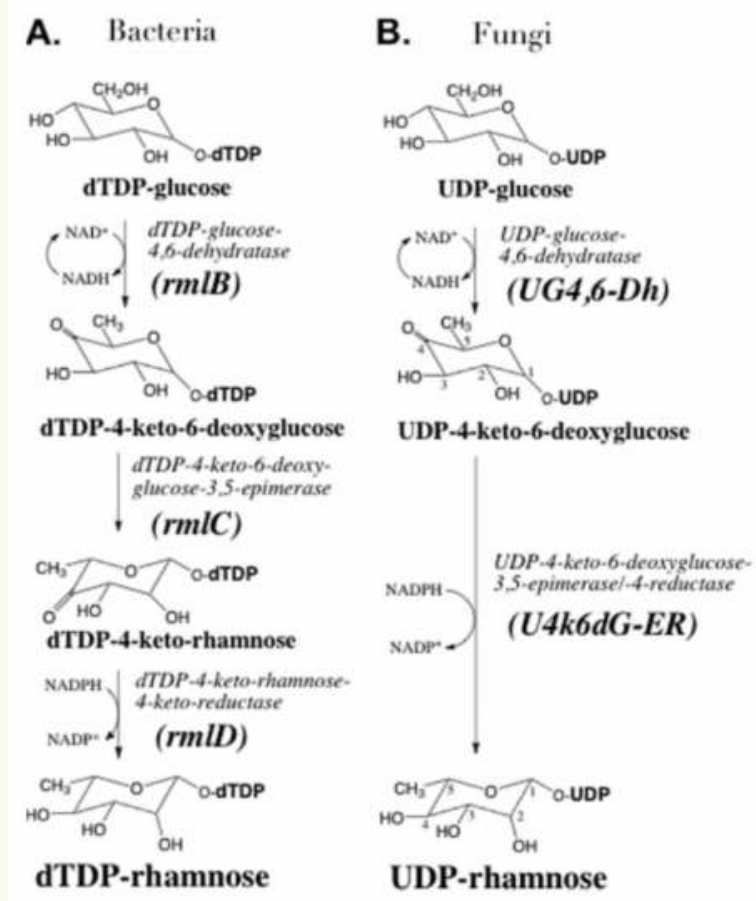

Figure 1.

In bacteria the final step of the synthesis is catalyzed by the RmID enzyme that converts dTDP-6-deoxy-L-xylo-4-hexulose to dTDP-L-rhamnose. Using bioinformatics approach we identified the putative orthologue of $R m I D$ in $S$. schenckii, which encodes for a 4-deoxyrhamnose reductase.

\section{AIM}

To elucidate the role of L-Rhamnose of the PRM present in the cell wall of $S$. schenckii on the interaction with the host immune system. To achieve this, we have three particular objectives: to silence the $S$. schenckii $\mathrm{rm} / \mathrm{D}$ gene, to characterize the $\mathrm{rm} / \mathrm{D}$ mutants and to determine the interaction of the $S$. schenckii mutant cells with the host

\section{CONCLUSIONS}

The cell wall PRM present of $S$. schenckii is recognized by the immune system of the host, in order to know if the Rhamnose present in these molecules is important for the interaction we silenced the gene rm/D involved in the synthesis of this carbohydrate. The mutants obtained show a 0.5 and 0.4 fold decrease in the expression of the gene. This decrease affects negatively the Rhamnose content in the cell wall, suggesting this gene is involved in the synthesis of L-Rhamnose. Further analysis is being made to see if these phenotypes are sufficient to expect a difference in the interaction of the fungus with the immune system.

\section{REFERENCES}

1. Lopes-Bezerra, L.M. 2011. Sporothrix schenckii Cell Wall Peptidorhamnomannans. Frontiers in microbiology. 2, (Jan. 2011), 243.

2. Mäki, M. and Renkonen, R. 2004. Biosynthesis of 6-deoxyhexose glycans in bacteria. Glycobiology. 14, 3 (2004), 1R-15R.

3. Lima, O.C. and Bezerra, L.M. 1997. Identification of a concanavalin A-binding antigen of the cell surface of Sporothrix schenckii. Journal of medical and veterinary mycology . 35, 3, 167-72

\section{ACKNOWLEDGMENT}

This work was supported by Consejo Nacional de Ciencia y Tecnología (ref. CB2011/166860; PDCPN2014-247109, and FC 2015-02-834), Universidad de Guanajuato (ref. 0087/13; ref. 1025/2016), and Red Temática Glicociencia en Salud (CONACYT-México) mutants show a 0.5 fold decrease in the expression of the gene (Fig. 2).

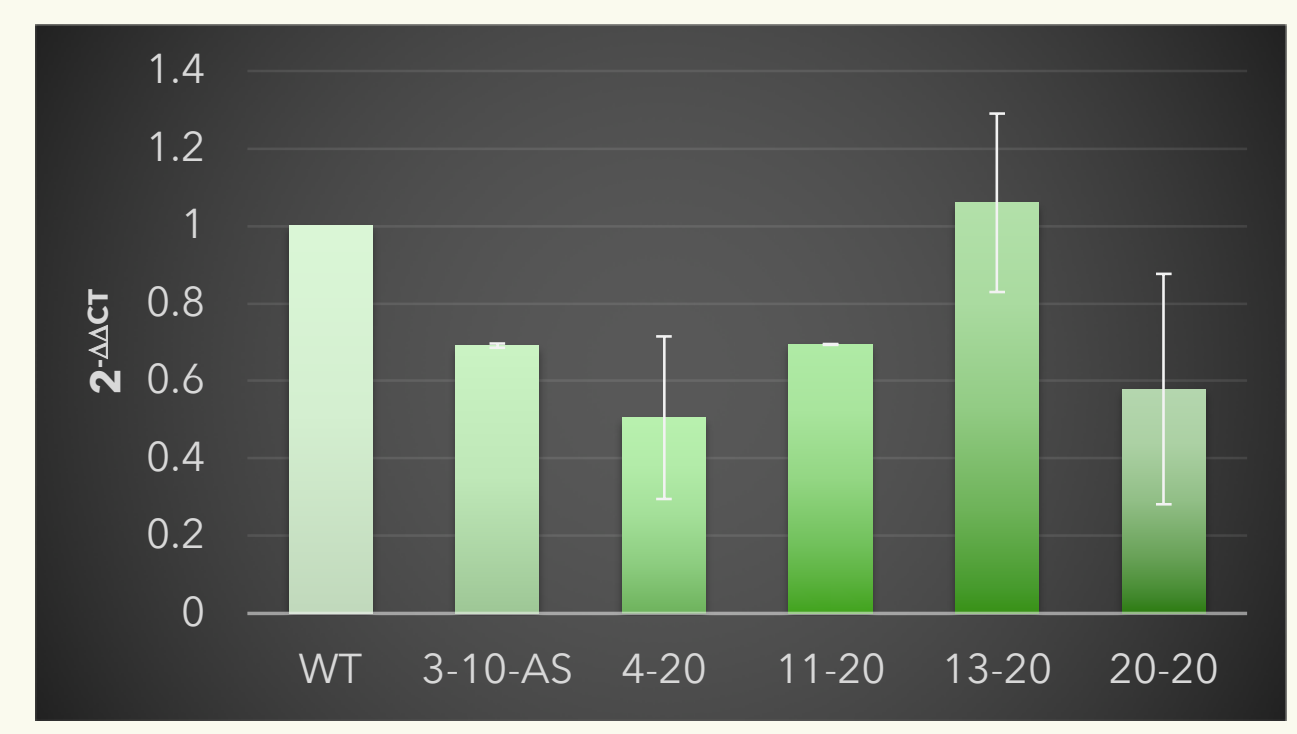

2. $r m / D$ gene expression. The relative level of expression of the $\mathrm{rm} / \mathrm{D}$ gene was messure by Real-Time PCR of the silenced mutants In order to know if the degree of silencing of the mutants we obtained is sufficient to have the expected phenotype, we evaluated some phenotypic characteristic like the binding of Alcian Blue dye to the cell wall (Fig. 3) and the cell wall content of Rhamnose in hyphae morphology (Fig. 4).

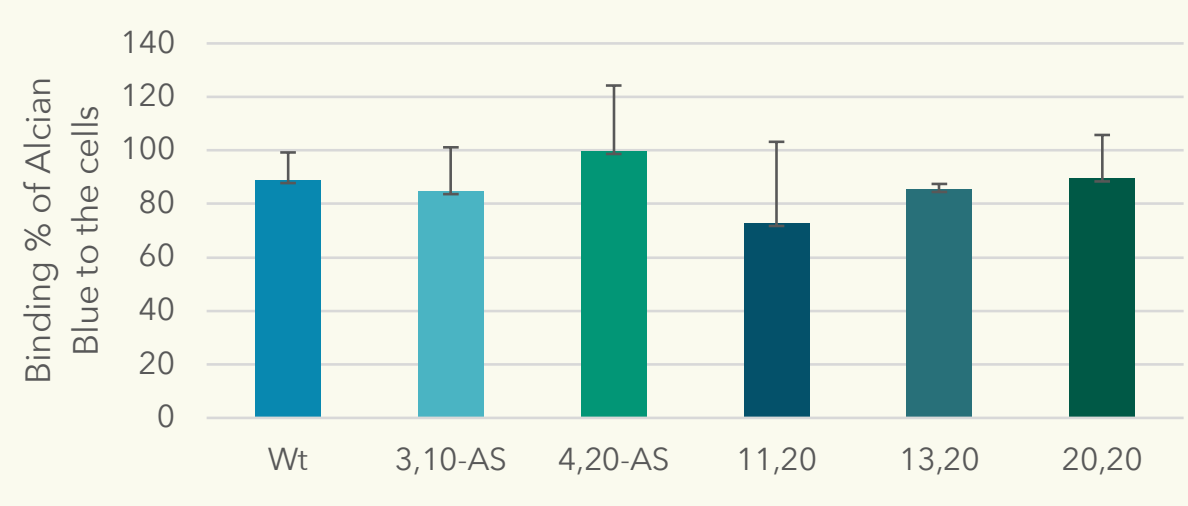

Figure 3. Alcian Blue Binding. The binding of the dye Alcian Blue to the cell wall changed. No one show a significative change.

Figure 4. Relative carbohydrate content of the cell wall. To know if the content of Rhamnose in the cell wall changed in the mutants, we measure the total carbohydrate content, two of the mutants show a light decrease in the content of Rhamnose. *Result of a single experiment. the cell wall of the mutants was measure to see if the overal contents of 